\title{
Validity of coupling impedance bench measurements
}

\author{
H. Hahn \\ Brookhaven National Laboratory, Upton, New York 11973-5000
}

(Received 7 September 2000; published 4 December 2000)

\begin{abstract}
In this paper the validity of coupling impedance bench measurements is theoretically demonstrated for the standard model of a uniformly distributed wall impedance. Integral equations expressing the magnetic field at the wall are found for the two cases where the device is excited either by a beam or a central wire. The integral equations are solved by a perturbation method with the coupling impedance as the expansion parameter, leading to expressions for the coupling impedance of the beam and the forward scattering coefficient in the bench measurement. Conditions for the validity of bench measurements are indicated, and the interpretation of wire measurements via the scattering coefficient by the conventional formulas is discussed.
\end{abstract}

PACS numbers: 29.27.-a, 29.27.Bd

\section{INTRODUCTION}

The electromagnetic interaction of a charged particle beam with its surroundings in the accelerator or collider is conveniently described by the coupling impedances of its components. The concept of coupling impedance apparently was originated by Vaccaro [1] and applied by Sessler and Vaccaro [2] to the analysis of the longitudinal, negative mass instability. Coupling impedance is basically an engineering concept, since it is defined as the ratio of voltage divided by current, and as such is amenable to established circuit theory. Ignoring the space charge term in the case of ultrarelativistic beams, the impedance is visualized either as a lumped element in a perfectly conducting beam tube or a beam tube section of finite length with a uniformly distributed wall impedance. The impedance can be frequency dependent but is usually assumed to be linear.

The general procedure to measure the longitudinal coupling impedance was developed for the LBL-ERA study by Faltens et al. by means of an analog in which the beam is replaced with a conductor [3]. Underlying this approach is the fact that the fields of an ultrarelativistic beam on the beam tube wall can be simulated by the propagation of a time-harmonic TEM mode in the transmission line so formed. An alternate method, not addressed in this paper, was suggested by Sands and Rees, according to which a short "delta" pulse is sent through the analog structure [4]. The coupling impedance is then obtained from the induced wake function by appropriate Fourier transform. Practical aspects of coupling impedance bench measurements and further references can be found in Caspers's review papers $[5,6]$.

Assessing the validity of coupling impedance bench measurements requires answers to two questions: first, what are the correct model and mathematical formulas for the interpretation of the experimental results and then, to what degree does this impedance value approximate the actual interaction with the beam. In spite of the experimental and theoretical work by many researchers, apparently no definite study has been presented. This paper attempts to answer these questions for the case of a lumped impedance as well as the general case represented by the model for a uniformly distributed impedance.

In the typical bench measurement involving a network analyzer, the forward scattering coefficient, $S_{21}$ (DUT), for the device under test (DUT) is obtained in the frequency domain and is compared to that for a reference section of equal length. In order to simplify the notation in this paper, the forward scattering coefficient of the reference line is assumed to be calibrated out, i.e., $S_{21}($ ref $)=1$. The normalized ratio, here simply taken as $S_{21}$, has been interpreted via standard transmission line circuit theory. The result for a lumped device, i.e., short compared to the beam tube radius, was given by Hahn and Pedersen (HP) as [7]

$$
Z_{\mathrm{HP}}=2 Z_{c} \frac{1-S_{21}}{S_{21}}
$$

with $Z_{c}$ the characteristic impedance of the reference line. In addition, their paper pointed out that the formula is also applicable to two or more separate lumped impedances, provided that they are small compared to the characteristic impedance. By extrapolation, the formula was suggested as a first approximation to a distributed impedance structure.

In the course of measuring distributed structures such as kickers, it was noticed that the HP formula can yield unphysical negative resistances. Using the Faltens model for distributed wall impedances, Walling et al. introduced the log formula for use in structures long compared to the beam tube diameter [8],

$$
Z_{\log }=-2 Z_{c} \ln S_{21} .
$$

This "standard" $\log$ formula is easy to use and represents a good approximation for a distributed impedance. Several "improved" impedance formulas have been suggested but are of marginal value, whereas the recent one by Jensen gives, under certain conditions, better results and deserves to be compared to the standard formula [9]. 
The justification for the bench measurement of the beam coupling impedance rests on the plausibility argument that the electromagnetic field of an ultrarelativistic beam is very similar to that produced by the coax in the limit of an infinitely thin wire. This reasoning was, to some extent, confirmed by Gluckstern's analysis of the effects of a wire on a resonant cavity [10]. In Gluckstern's paper, integral expressions for the beam impedance and the scattering coefficient due to the cavity impedance are given, based on which the equality of the impedances in the thin wire limit is suggested. The paucity of detailed results in this paper pointed to the need for further studies. In the present paper, Gluckstern's procedure is applied to the model of a lumped as well as a distributed wall impedance, resulting in integral equations for the two cases of beam and wire. The integral equation for the lumped case can be solved directly, whereas the solution for the distributed case is obtained by a perturbation method with the coupling impedance as expansion parameter. Solving the integral equations leads in both cases to explicit expressions for the coupling impedance seen by the beam and the forward scattering coefficient in the virtual bench measurement. The theoretical scattering coefficients are interpreted via the above impedance formulas and the resulting impedance values are then compared with those obtained directly for the beam. This comparison permits one to establish the requirements for valid coupling impedance bench measurements and provides for an error estimate of the experimental results.

\section{INTEGRAL EQUATION}

The topic considered in this paper can be defined by an integral equation for the magnetic field generated by an ultrarelativistic beam or a coaxial central conductor in the case of bench measurements. The azimuthal component of the magnetic field at the wall is given by [11] (in natural units $c=1, \mu_{0}=1$, and $Z_{0}=c \mu_{0}=1$ )

$$
H_{\varphi}=\frac{1}{2 \pi b} e^{-j k z}-j \frac{k b}{2 \pi} \int d z^{\prime} E_{z}\left(b, z^{\prime}\right) K_{p}(u),
$$

with $K_{p}(u)$ the kernel appropriate for the pipe region, either empty or with a central conductor, and $u=\left|z-z^{\prime}\right|$.

The coupling impedance, $R$, is in the case of a distributed impedance assumed to be caused by a wall impedance, $R_{s q}$, uniformly distributed over a length, $g$, in an infinitely long beam pipe of radius, $b$,

$$
R=\frac{g}{2 \pi b} R_{s q} .
$$

The wall current, associated with the azimuthal magnetic field, produces a longitudinal electric field,

$$
E_{z}(b, z)=-R_{s q} H_{\varphi}(b, z) .
$$

The integral equation, Eq. (3), thus, can be rewritten as

$$
H_{\varphi}=\frac{I}{2 \pi b} e^{-j k z}+j k \frac{b^{2}}{g} R \int_{0}^{g} d z^{\prime} H_{\varphi}\left(b, z^{\prime}\right) K_{p}(u),
$$

which is solved by a perturbation method, where $R$ (i.e., $R / Z_{0}$ in SI units) is taken as the perturbation parameter. In this vein, the magnetic field is expanded as

$$
H_{\varphi}(b, z)=F_{0}(z)+R F_{1}(z)+R^{2} F_{2}(z)+\cdots,
$$

with

$$
F_{0}=\frac{I}{2 \pi b} e^{-j k z}
$$

and the higher order terms obtained by iteration according to

$$
F_{i+1}=-j k \frac{b^{2}}{g} \int_{0}^{g} d z^{\prime} F_{i}\left(z^{\prime}\right) K_{p}(u) .
$$

On the other hand, the case of a lumped impedance leads to the integral equation

$$
H_{\varphi}=\frac{I}{2 \pi b} e^{-j k z}-j \frac{k b}{2 \pi} R I \int d z^{\prime} \delta\left(z^{\prime}\right) K_{p}(u),
$$

for which a solution exists which is valid for all values of $R$.

\section{ANALYSIS FOR THE BEAM}

The solution of the integral equation in the case of a beam is obtained via the kernel for the pipe region. The full expression for the pipe kernel assumes lossless boundaries whereas in real cases fields above the tube cutoff are damped and can be ignored. Thus, for the purpose of the present study, the kernel is limited to the terms below cutoff,

$$
K_{p}=-\frac{2 \pi}{b^{2}} \sum_{1}^{n c} \frac{e^{-\Lambda_{n} u}}{\Lambda_{n}}
$$

with

$$
\Lambda_{n}=\frac{j_{0 n}}{b} \sqrt{1-\left(k b / j_{0 n}\right)^{2}}
$$

and $j_{0 n}$ the zeros of the Bessel function $J_{0}\left(j_{0 n}\right)=0$.

The coupling impedance seen by the beam is defined as

$$
\begin{aligned}
Z & =-\frac{1}{I} \int_{0}^{g} E_{z}(b, z) e^{j k z} d z \\
& =R+\frac{R^{2}}{I} \int_{0}^{g} F_{1}(z) e^{j k z} d z+\cdots
\end{aligned}
$$

After lengthy but straightforward integrations, one finds the coupling impedance to second order in $R$,

$$
Z=R+j 2 \frac{k b}{g} R R_{s q} \sum_{n} \frac{1}{j_{0 n}^{4} \Lambda_{n}}\left\{j_{0 n}^{2}\left(\Lambda_{n} g-1\right)+2 k^{2} b^{2}-\left[2 b^{2} k \Lambda_{n} \sin \Theta+\left(2 k^{2} b^{2}-j_{0 n}^{2}\right) \cos \Theta\right] e^{-\Lambda_{n} g}\right\},
$$

with the electrical length of the impedance $\Theta=k g$. 


\section{A. Low-frequency approximation}

Of particular interest is the low-frequency limit, $k b \leq 1$, where $\Lambda_{n} \approx j_{0 n} / b$ and

$$
\begin{aligned}
Z \approx R\left[1+j 2 k \frac{b^{2}}{g} R_{s q} \sum_{n} \frac{1}{j_{0 n}^{3}}(\right. & j_{0 n} g / b-1 \\
& \left.\left.+\cos \Theta e^{-j_{0 n} g / b}\right)\right] .
\end{aligned}
$$

This result suggests that the beam sees the wall impedance, $R$, with the addition of end effects, the latter depending on the strength of the perturbation, $R_{s q}$.

\section{B. Lumped impedance}

One approximation further, by taking the limit $g \rightarrow 0$, one finds the expression for a lumped impedance:

$$
Z \approx R\left(1+j 2 \pi k b R \sum_{n} \frac{1}{j_{0 n}}\right) .
$$

Note that the end effects in the case of the lumped impedance depend on the coupling impedance itself rather than $R_{s q}$, which here is only a mathematical tool.

This expression for the lumped impedance can be obtained directly from the integral equation, Eq. (9), which is valid for all values of $R$. This correspondence allows one to infer that the other results for the coupling impedance seen by the beam remain generally valid also.

\section{ANALYSIS FOR THE WIRE MEASUREMENT}

Bench measurements are performed in order to experimentally determine the forward scattering coefficient by inserting a wire into the device, thus transforming it into a coaxial transmission line with outer and inner radii, $b$ and $a$, respectively. The analysis objective in the case of a wire measurement is to find a theoretical expression for this coefficient, which is defined as

$$
S_{21}=H_{\varphi}(b,+\infty) / \frac{I}{2 \pi b} e^{-j k z}
$$

The solution of the integral equation for the magnetic field is obtained in full analogy to that for the beam, but now with the kernel given by

$$
K_{p}(u)=-j \frac{1}{2 k b^{2} Z_{c}} e^{-j k u}-\frac{2 \pi}{b^{2}} \sum_{1}^{n c} \frac{\alpha_{n}}{\lambda_{n}} e^{-\lambda_{n} u},
$$

where the characteristic impedance of the coaxial structure is (in natural units)

$$
Z_{c}=\frac{1}{2 \pi} \ln \frac{b}{a},
$$

and the other kernel quantities are given by

$$
\begin{gathered}
\lambda_{n}=\frac{i_{0 n}}{b} \sqrt{1-\left(k b / i_{0 n}\right)^{2}}, \\
\alpha_{n}=\frac{J_{0}^{2}\left(i_{0 n} a / b\right)}{J_{0}^{2}\left(i_{0 n} a / b\right)-J_{0}^{2}\left(i_{0 n}\right)},
\end{gathered}
$$

with $i_{0 n}$ being the zero solutions of

$$
J_{0}\left(i_{0 n} a / b\right) Y_{0}\left(i_{0 n}\right)=Y_{0}\left(i_{0 n} a / b\right) J_{0}\left(i_{0 n}\right) .
$$

The theoretical expression for the forward scattering coefficient follows after some manipulations as

$$
\begin{aligned}
S_{21}= & 1-\frac{R}{2 Z_{c}}+\frac{1}{2}\left(\frac{R}{2 Z_{c}}\right)^{2}\left\{1-j \frac{1}{\Theta}+\frac{1}{\Theta^{2}} e^{-j \Theta}\left(1-e^{-j \Theta}\right)\right\} \\
& -j 2 \frac{k b}{g}\left(\frac{R}{2 Z_{c}}\right) R_{s q} \sum_{1}^{n c} \frac{\alpha_{n}}{i_{0 n}^{4} \lambda_{n}}\left\{i_{0 n}^{2}\left(\lambda_{n} g-1\right)+2 k^{2} b^{2}-\left[2 k b^{2} \lambda_{n} \sin \Theta+\left(2 k^{2} b^{2}-i_{0 n}^{2}\right) \cos \Theta\right]\right\} .
\end{aligned}
$$

\section{A. Low-frequency approximation}

Bench measurements, and certainly the present paper, implicitly assume that the validity of the results is limited to the frequency range below cutoff where $k b \ll 1$, even though the electrical length $\Theta=k g$ can be finite. The scattering coefficient in the low-frequency limit simply follows from Eq. (19) by taking $\lambda_{n} \approx i_{0 n} / b$, resulting in

$$
S_{21}=1-\frac{R}{2 Z_{c}}\left\{1-\frac{R}{4 Z_{c}}\left[1-j \frac{1}{\Theta}+\frac{1}{\Theta^{2}}\left(1-e^{-j \Theta}\right) e^{-j \Theta}\right]+2 j \frac{k b^{2}}{g} R_{s q} \sum_{n} \frac{\alpha_{n}}{i_{0 n}^{3}}\left(i_{0 n} g / b-1+\cos \Theta e^{-i_{0 n} g / b}\right)\right\} .
$$

\section{B. Thin wire approximation}

It is well known that wire measurements require the use of the thinnest possible wire in order to obtain meaningful coupling impedance results. Reducing the wire however is limited both by mechanical and electrical constraints, the latter because the mismatch between the characteristic impedance of the reference line and the standard $50 \Omega$ of the network analyzer becomes increasingly difficult to correct. In the thin wire limit one finds 


$$
i_{0 n}=j_{0 n}+\varepsilon_{n} \approx j_{0 n}+\frac{\pi Y_{0}\left(j_{0 n}\right)}{2 J_{1}\left(j_{0 n}\right)}\left[1 / \ln \left(\frac{2 b}{\gamma j_{0 n} a}\right)\right],
$$

with $\ln \gamma \approx 0.577$ being Euler's constant. It follows that

$$
\alpha_{n} \approx 1+J_{1}^{2}\left(j_{0 n}\right) \varepsilon_{n}^{2} .
$$

For the typical case of $b / a=100$ one finds $\varepsilon_{n} \approx 0.41$ and $\alpha_{n} \approx 1.05$. It should be noted that in the thin wire approximation the relative contribution from the end effects in the expression for the beam impedance and the forward scattering coefficient approach equality but only logarithmically - differing in the typical case by less than a factor of 2 . This fact justifies combining the straight tube impedance, $R$, with the end effects into a total impedance value, $Z$. For the sake of simplicity, the sequel of this paper will assume the thin wire approximation. The thin wire expression for the forward scattering coefficient now takes the form

$$
\begin{aligned}
S_{21} \approx 1-\frac{Z}{2 Z_{c}}\left\{1-\frac{Z}{4 Z_{c}}\right. & {\left[1-j \frac{1}{\Theta}+\frac{1}{\Theta^{2}}\right.} \\
& \left.\left.\times\left(1-e^{-j \Theta}\right) e^{-j \Theta}\right]\right\} .
\end{aligned}
$$

\section{Lumped impedance}

Correspondingly, the scattering coefficient for a lumped impedance follows as

$$
S_{21} \approx 1-\frac{Z}{2 Z_{c}}+\frac{5}{4}\left(\frac{Z}{2 Z_{c}}\right)^{2} .
$$

\section{TRANSMISSION LINE ANALYSIS}

The standard formulas used to interpret coupling impedance bench measurements were all derived in the framework of transmission line theory. The field configuration on an ideal transmission line is a TEM wave with purely transverse components. A finite wall conductivity changes the field into a mode with an axial component of the electric field. The assumption in the transmission line theory is, however, that the analysis can be performed with ideal walls and the real situation is handled by appropriately modifying the characteristic impedance and propagation constant. Terminal effects, i.e., the local appearance of evanescent modes, for example, at the junction of two different transmission lines, is also considered to be negligibly small. End effects can to some degree be represented by added capacitive elements [12]. It is also plausible that the relative contribution of end effects is smaller for distributed impedances than for lumped impedances. For the purpose of coupling impedance, it is thus suggested that bench measurements should be performed on devices with beam tubes attached as part of the unit. Notwithstanding its limitations, transmission line analysis represents a powerful tool and its results must be compared with the field analysis in the present paper.

\section{A. Distributed impedance}

The transmission line analysis of a distributed impedance can be based on the Faltens model although it only represents an approximation valid for small wall impedance values. In this model the characteristic impedance and propagation constant of the DUT are defined as [3]

$$
Z_{\text {DUT }}=Z_{c} \sqrt{1-j \frac{Z}{\Theta Z_{c}}}=\eta Z_{c},
$$

and

$$
k_{\mathrm{DUT}}=k \sqrt{1-j \frac{Z}{\Theta Z_{c}}}=\eta k .
$$

Representing the amplitude of the forward and reflected wave by $A$ and $B$, respectively, one can apply field matching (i.e., voltage and current matching in the transmission line) which leads to the conditions at the input port

$$
\begin{gathered}
A_{\mathrm{in}}+B_{\mathrm{in}}=A_{\mathrm{DUT}}+B_{\mathrm{DUT}}, \\
\left(A_{\mathrm{in}}-B_{\mathrm{in}}\right)=\frac{1}{\eta}\left(A_{\mathrm{DUT}}+B_{\mathrm{DUT}}\right),
\end{gathered}
$$

and at the output port

$$
\begin{gathered}
A_{\mathrm{DUT}} e^{-j \Theta_{\mathrm{DUT}}}+B_{\mathrm{DUT}} e^{-j \Theta_{\mathrm{DUT}}}=B_{\mathrm{out}}, \\
\frac{1}{\eta}\left(A_{\mathrm{DUT}} e^{-J \Theta_{\mathrm{DUT}}}-B_{\mathrm{DUT}} e^{-j \Theta_{\mathrm{DUT}}}\right)=B_{\mathrm{out}} .
\end{gathered}
$$

With the scattering coefficient defined as

$$
S_{21}^{\mathrm{TEM}}=\frac{B_{\text {out }}}{A_{\text {in }} e^{-j \Theta}},
$$

one finds after simple manipulations [13]

$$
S_{21}^{\mathrm{TEM}}=\frac{4 \eta e^{-(\eta-1) \Theta}}{(\eta+1)^{2}-(\eta-1)^{2} e^{-j 2 \eta \Theta}} .
$$

Taylor expansion with regard to the coupling impedance, $Z$, leads to

$$
\begin{aligned}
S_{21}^{\mathrm{TEM}}=1-\frac{Z}{2 Z_{c}}\left\{1-\frac{Z}{4 Z_{c}}\right. & {\left[1-\frac{j}{\Theta}+\frac{1}{2 \Theta^{2}}\right.} \\
& \left.\left.\times\left(1-e^{-j 2 \Theta}\right)\right]\right\}+\cdots .
\end{aligned}
$$

A first order approximation to Eq. (28) leads to Walling's $\log$ formula [8], Eq. (2),

$$
Z_{\log }=-2 Z_{c} \ln S_{21} \text {. }
$$

Recently, Jensen proposed an improved log formula for a distributed impedance [9]

$$
Z_{\mathrm{Log}}=-2 Z_{c} \ln S_{21}\left(1+j \frac{\ln S_{21}}{2 \Theta}\right) .
$$




\section{B. Lumped impedance}

In the limit of $\Theta \rightarrow 0$, the distributed impedance turns into a lumped impedance with

$$
S_{21}^{\mathrm{TEM}}=1-\frac{Z}{2 Z_{c}}+\left(\frac{Z}{2 Z_{c}}\right)^{2}+\cdots,
$$

which to second order is in agreement with the expression obtained from the lumped impedance formula, Eq. (1),

$$
S_{21}^{\mathrm{TEM}}=\frac{2 Z_{c}}{2 Z_{c}-Z} \approx 1-\frac{Z}{2 Z_{c}}+\left(\frac{Z}{2 Z_{c}}\right)^{2}+\cdots .
$$

The lumped impedance formula is derived in the framework of standard transmission line theory and therefore is valid for all impedance values. In view of this agreement, one can infer that the expressions for the distributed impedance are also more broadly valid even though the Faltens model is only approximate.

Note that the second order difference between transmission line and field analysis for the lumped impedance is

$$
S_{21}^{\mathrm{TEM}}-S_{21}=-\frac{1}{5}\left(\frac{Z}{2 Z_{c}}\right)^{2}
$$

which is a consequence of neglecting evanescent modes in the transmission line analysis.

\section{INTERPRETATION OF BENCH MEASUREMENTS}

The above discussion suggests that for lumped impedances, i.e., $\Theta \leq 1$, bench measurements can be used to determine the coupling impedance seen by the beam, if certain precautions are taken and the scattering coefficient is interpreted via the HP-lumped impedance formula.

The interpretation of bench measurements on distributed structures, i.e., $\Theta \geq 1$, requires the $\log$ formula, the validity of which requires further discussion. The field analysis results presented in this paper are claimed to represent a more accurate description of the coupling impedance bench measurements than that obtained from the standard transmission line treatment. However, the formulas are complex and do not lead to explicit expressions for the coupling impedance. On the other hand, they can serve to estimate the error made by using the standard formulas. Although the analysis was based on structures with uniformly distributed wall impedance, the conclusions are expected to remain valid for reasonably nonuniform distributions also.

Applying the appropriate field analysis scattering coefficient, Eq. (23), to the standard impedance formulas yields the following expressions for the systematic error in the Hahn-Pedersen lumped impedance formula, Eq. (1),

$$
\frac{Z_{\mathrm{HP}}}{Z}=1+\frac{Z}{4 Z_{c}}\left(1+\frac{j}{\Theta}-\frac{\left(1-e^{-j \Theta}\right) e^{-j \Theta}}{\Theta^{2}}\right),
$$

the Walling et al. $\log$ formula, Eq. (2),

$$
\frac{Z_{\log }}{Z}=1+\frac{Z}{4 Z_{c}}\left(\frac{j}{\Theta}-\frac{\left(j-e^{-j \Theta}\right) e^{-j \Theta}}{\Theta^{2}}\right),
$$

and the Jensen improved log formula, Eq. (29),

$$
\frac{Z_{\mathrm{Log}}}{Z}=1-\frac{Z}{4 Z_{c}} \frac{\left(1-e^{-j \Theta}\right) e^{-j \Theta}}{\Theta^{2}} .
$$

The error analysis confirms the advantage of using Walling's $\log$ formula for distributed impedances rather than the HP formula and shows the further improvement in Jensen's log formula. However, the improved log formula is restricted to long structures with $\Theta \geq 1$, whereas the regular log formula could be used for lumped impedances, albeit with reduced accuracy.

In summary, it can be stated that the present analysis in all cases confirms the validity of coupling impedance bench measurements well below cutoff, provided that the wire size is made sufficiently small and the measurement is performed with attached beam tubes of sufficient length in order to avoid the effects due to evanescent modes.

[1] V. G. Vaccaro, CERN-ISR-RF 66-35, 1966.

[2] A. Sessler and V. Vaccaro, CERN-67-2, 1967.

[3] A. Faltens, E. C. Hartwig, D. Mohl, and A. M. Sessler, in Proceedings of the 8th International Conference on High-Energy Accelerators, 1971, edited by M. H. Blewett (CERN, Geneva, 1971), p. 338.

[4] M. Sands and J. Rees, PEP-95, 1974.

[5] F. Caspers, in Lecture Notes in Physics, edited by M. Dienes, M. Month, S. Turner (Springer-Verlag, Berlin, 1992), No. 400, p. 80.

[6] F. Caspers, in Handbook of Accelerator Physics and Engineering, edited by A. W. Chao and M. Tigner (World Scientific, Singapore, 1998), p. 570.

[7] H. Hahn and F. Pedersen, BNL50870, 1978.

[8] L. S. Walling, D. E. McMurray, D. V. Neuffer, and H. A. Thiessen, Nucl. Instrum. Methods Phys. Res., Sect. A 281, 433 (1989).

[9] E. Jensen, PS/RF/Note 2000-001, 2000.

[10] R. L. Gluckstern and R. Li, Part. Accel. 29, 159 (1990).

[11] B. W. Zotter and A. K. Kheifets, Impedances and Wakes in High-Energy Particle Accelerators (World Scientific, Singapore, 1998), p. 237.

[12] R.W.P. King, in Encyclopedia of Physics, edited by S. Fluegge (Springer-Verlag, Berlin, 1958), Vol. XVI, Chap. 17, p. 210.

[13] H. Hahn, M. Morvillo, and A. Ratti, AD/RHIC/RD-95, 1995. 